\title{
O impacto do nível socioeconómico na acessibilidade aos cuidados de saúde primários: estudo em quatro unidades de saúde do Norte de Portugal
}

Vânia Teixeira, ${ }^{1}$ Ana Mafalda Macedo, ${ }^{2}$ Catarina Borges, ${ }^{3}$ Sofia Carrapa, ${ }^{3}$ Sara China, ${ }^{3}$ Raquel Cunha ${ }^{4}$

\section{RESUMO}

Objetivos: Relacionar o nível socioeconómico com a acessibilidade aos cuidados de saúde primários em quatro Unidades de Saúde Familiar (USF), nas dimensões que surgem após a perceção, por parte dos utentes, da necessidade de cuidados de saúde: capacidade e acomodação, esforço financeiro e adequabilidade.

Tipo de Estudo: Estudo observacional descritivo, transversal e analítico.

Local: USF Brás-Oleiro, USF São Pedro da Cova, USF Bracara Augusta e USF Prelada.

População: Utentes com idade igual ou superior a 18 anos, inscritos nas USFs supracitadas há mais de doze meses, pertencentes às listas dos orientadores de formação das investigadoras e com pelo menos um contacto com a sua USF nos últimos 24 meses.

Métodos: Estudo realizado entre abril e dezembro de 2014 através da aplicação de um questionário por via telefónica. Cada uma das dimensões da acessibilidade foi avaliada através de um score global que foi posteriormente relacionado com o nível socioeconómico da população em estudo. Foram utilizados os testes estatísticos do Qui-Quadrado, Mann-Whitney, Kruskal-Wallis e a correlação de Spearman. Resultados: Dos 1.250 utentes selecionados, $692(55,4 \%)$ responderam ao questionário. Verificou-se existirem diferenças estatisticamente significativas entre as USF relativamente a cada score $(p<0,05)$. A escolaridade, o rendimento, o número de elementos do agregado familiar e a situação de isenção apresentaram correlação estatisticamente significativa com todos os scores. Constatou-se que a idade, a situação profissional, o estado geral de saúde e a duração da relação do utente com o seu médico de família se correlacionaram de forma estatisticamente significativa com os scores «Capacidade e Acomodação» $(p<0,05)$ e «Adequabilidade» $(p<0,05)$.

Conclusões: Globalmente, o presente estudo permitiu inferir que, para a população analisada, um nível socioeconómico mais desfavorecido e uma pior perceção do estado de saúde se correlacionaram com uma maior acessibilidade, fundamentalmente no que diz respeito à «Capacidade e Acomodação» e «Adequabilidade».

Palavras-chave: Cuidados de Saúde Primários; Nível Socioeconómico; Acessibilidade.

\section{INTRODUÇÃO}

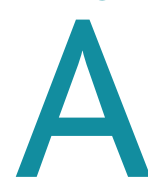

Declaração de Alma-Ata considerou os cuidados de saúde primários (CSP) a solução para o alcance de um nível de saúde que permita a todos uma vida económica e socialmente produtiva, representando o primeiro ponto de contacto dos indivíduos, da família e da comunidade com o sistema de saúde. ${ }^{1}$

Os serviços de saúde são determinantes importantes do bem-estar da população e a desigualdade à sua acessibilidade e utilização podem contribuir para as assimetrias na saúde. Num sistema de saúde ideal, um serviço equitativo é aquele em que o acesso aos cuidados de saúde está associado às necessidades independentemente da capa-

'Médica assistente de Medicina Geral e Familiar, Unidade de Cuidados Continuados, Hospital-escola Universidade Fernando Pessoa

${ }^{2}$ Médica assistente de Medicina Geral e Familiar, UCSP Cinfães -ACeS Baixo Tâmega 3Médica assistente de Medicina Geral e Familiar, UCSP santo António do Laranjeiro, ACeS Almada -Seixal

${ }^{4}$ Médica assistente de Medicina Geral e Familiar, UCSP Castro D'Aire, ACeS Dão Lafões ${ }_{5}^{5}$ Médica assistente de Medicina Geral e Familiar, Centro de Saúde da Marinha Grande - ACeS Pinhal Litoral

${ }^{6}$ Médica assistente de Medicina Geral e Familiar, UCSP Terras de Bouro, ACeS Gerês - Cabreira 
cidade para pagar. ${ }^{2}$ Existe evidência de que os indivíduos com nível socioeconómico mais baixo são mais doentes e, neste contexto, tendem a utilizar mais os serviços de saúde mas, em contraste, recorrem menos a serviços especializados do que os indivíduos com nível socioeconómico mais elevado. ${ }^{3}$ Em Portugal foram descritas menos visitas ao médico de família (MF) por indivíduos pertencentes a grupos socioeconómicos mais baixos. ${ }^{2}$

A acessibilidade tem sido idealizada de diversas formas. O modelo de acessibilidade desenvolvido por Pechansky e Thomas ${ }^{4}$ assenta no conceito de ajuste entre a capacidade do sistema de saúde e a possibilidade ou a facilidade com a qual os utentes ou as populações são capazes de usar os serviços adequados às suas necessidades.

Um estudo recente ${ }^{5}$ conceptualizou que o acesso pode ser medido pela avaliação das seguintes dimensões: proximidade (approachability), aceitabilidade (acceptability), capacidade e acomodação (availability and accomodation), esforço financeiro (affordability) e adequabilidade (appropriateness).

A proximidade é caracterizada pela capacidade das pessoas que necessitam de cuidados de saúde conseguirem aferir que alguma forma de serviços existe, pode ser usada e tem um impacto sobre a saúde destas. A noção da capacidade de perceber a necessidade de cuidados entre as populações é crucial e determinada por fatores como a literacia em saúde, o conhecimento sobre a saúde e crenças relacionadas com a saúde e a doença.

A aceitabilidade diz respeito a fatores culturais e sociais, determinando a possibilidade das pessoas aceitarem os aspetos do serviço (e.g., o género ou o grupo social dos prestadores de cuidados) e a adequabilidade do discernimento para as pessoas procurarem os serviços.

Já a capacidade e acomodação representam a medida em que o prestador detém os recursos necessários como pessoal e tecnologia - para atender às necessidades dos utentes. Este traduz uma medição da capacidade da rede em oferecer serviços em quantidade suficiente para fazer face à procura potencial. À luz desta dimensão avalia-se se a organização do prestador (em termos de horários de funcionamento, marcação de consultas, etc.) é adequada às restrições e preferências dos utentes.

O esforço financeiro refere-se à capacidade económica ou à disposição de tempo por parte dos utentes para suportar os encargos com a prestação de cuidados de saúde. É o resultado direto dos preços dos serviços e despesas relacionadas, além dos custos de oportunidade relacionados com a perda de remuneração durante o tempo que precisam dos serviços de saúde. Pobreza, isolamento social ou endividamento são exemplos de fatores que restringem a capacidade das pessoas para pagar os cuidados necessários.

A adequabilidade denota o ajuste entre os serviços disponíveis e o que os utentes precisam, a quantidade de tempo gasto na avaliação dos problemas de saúde e na determinação do tratamento correto e a qualidade técnica e interpessoal dos serviços prestados. Esta proporcionalidade relaciona-se com a adequabilidade (quais os serviços prestados) e a qualidade (a forma como são fornecidos) dos serviços de saúde e a sua natureza integrada e contínua.

As várias dimensões da acessibilidade não são completamente independentes. Estas influenciam-se mutuamente e atuam a partir da interação de determinantes relacionados com as características dos indivíduos (lugar onde vivem, seus recursos económicos e estrato social) e dos serviços (quantidade, localização das instalações e custos).

Resumindo, o papel dos custos (entenda-se, não só os financeiros) do utente no Sistema Nacional de Saúde e a influência do nível socioeconómico das populações são áreas bastante sensíveis que necessitam de mais investigação.

O conhecimento de eventuais desigualdades no acesso a cuidados de saúde é fundamental. Assim, de acordo com o princípio de justiça distributiva, será de almejar um sistema de saúde em que o fator primordial, moderando o seu acesso, seja a necessidade de cuidados e não uma qualquer outra característica do indivíduo que o procure. Neste contexto, e por ser um tema de interesse das autoras, decidiram explorá-lo mais aprofundadamente nas populações que servem. Assim, pretende-se com este estudo verificar se o nível socioeconómico tem influência na acessibilidade aos CSP em quatro unidades selecionadas por conveniência - duas Unidades de Saúde Familiar (USF) do ACeS Grande Porto II-Gondomar, uma USF do ACeS Cávado I-Braga e uma USF do ACeS do Grande Porto V, Porto Ocidental-nas dimensões que surgem após a perceção, por parte dos utentes, da necessidade de cuidados de saúde: capacidade e acomodação, esforço financeiro e adequabilidade. Pretende-se, ainda, enfatizar as assimetrias que se venham a detetar nas unidades de saúde supracitadas.

\section{MÉTODOS}

Foi realizado um estudo observacional descritivo, 
transversal e analítico, entre abril e dezembro de 2014, nas USF Brás-Oleiro, São Pedro da Cova, Bracara Augusta e Prelada, através da aplicação de um questionário por via telefónica.

A população estudada contemplou utentes com idade igual ou superior a 18 anos, inscritos nas USF supracitadas há mais de 12 meses, pertencentes às listas dos orientadores de formação (OF) das investigadoras, com pelo menos um contacto com a sua USF nos últimos 24 meses e sem demência codificada no processo clínico (código P70, segundo a ICPC-2, International Classification of Primary Care, $2^{\text {nd }}$ edition).

Através do SIARS ${ }^{\circledR}$ e do MIMUF® apurou-se, no mês de novembro de 2013, uma população total de 7.753 utentes que preenchiam os critérios de inclusão. Considerando-se uma prevalência de 50\%, o tamanho amostral mínimo foi calculado em 938 utentes, de forma a poder estimar os resultados com margem de erro de $3 \% \mathrm{e}$ intervalos de confiança a 95\%. Prevendo uma taxa de recusa de participação de $30 \%$, as autoras aumentaram o tamanho amostral para 1.250 utentes, selecionados por processo de amostragem aleatória simples (recorreu-se ao programa Random Integer Generator, disponível em www.random.org). O número de questionários por investigadora obedeceu ao peso relativo que cada uma das listas de utentes tinha relativamente à população total apurada. De notar que, das seis investigadoras, três pertenciam à USF Brás-Oleiro, pelo que foram selecionados utentes de três listas diferentes. À USF São Pedro da Cova, Bracara Augusta e Prelada pertencia apenas uma investigadora e, como tal, foram selecionados utentes de apenas uma lista de cada uma das USF.

O questionário aplicado foi elaborado a partir do questionário do Primary Care Assessment Survey, ${ }^{6}$ facultado pelo próprio autor após a sua solicitação. As questões selecionadas foram aquelas que melhor se enquadravam ao objetivo deste estudo, tendo sido posteriormente adaptadas à língua portuguesa e elaboradas de forma a facilitar a sua aplicação via telefónica. Apesar de baseado no Primary Care Assessment Survey, o questionário aplicado foi inteiramente criado pelas investigadoras, não consistindo na tradução do questionário referido. Este era composto por nove questões relativas à identificação pessoal e caracterização do agregado familiar e dez questões sobre a opinião pessoal acerca do MF e Unidade de Saúde (US). Os utentes selecionados aleatoriamente foram, numa primeira fase, contactados via tele- fónica pelos administrativos de cada uma das USF correspondentes. Neste primeiro contacto, o secretário clínico/administrativo, seguindo um guião, explicava brevemente ao utente em que consistia o estudo para o qual tinha sido selecionado e que, caso aceitasse participar, seria posteriormente contactado por um médico da sua USF para responder ao questionário. Os contactos telefónicos foram efetuados das 8 às 20 horas. $\mathrm{Na}$ ausência de resposta ao primeiro contacto, quer pelo secretário clínico/administrativo na primeira fase, quer pelo investigador na segunda fase, eram efetuados, pelo menos, mais quatro tentativas de contacto posteriores, sempre em horários e dias diferentes. A todos os utentes que aceitaram participar foi garantida a confidencialidade das suas respostas e a liberdade de, a qualquer momento, desistirem de participar no estudo. Os utentes que não atenderam a chamada telefónica, os que se recusaram participar no estudo e os que ao longo da entrevista telefónica apresentaram evidente incapacidade/dificuldade em perceber as perguntas que lhes foram colocadas ou responderam de forma inapropriada foram excluídos. Destes, recolheu-se, do processo clínico, apenas o género e a idade. Os dados obtidos foram codificados e introduzidos em base de dados construída para o efeito, com recurso ao programa Microsoft Office Excel $2010^{\circledast}$.

Relativamente aos dados recolhidos foram definidas variáveis qualitativas (isenção, género, estado civil, situação profissional, profissão atual, rendimento mensal líquido do agregado familiar e estado geral de saúde) e quantitativas (idade, anos de escolaridade, número de elementos do agregado familiar e duração da relação do utente com o seu MF atual). Para cada uma das dimensões da acessibilidade («Capacidade e Acomodação», «Esforço Financeiro» e «Adequabilidade») foram definidas três variáveis qualitativas. $\mathrm{Na}$ avaliação da «Capacidade e Acomodação», as variáveis foram a «Facilidade de deslocação do utente à sua US», a «Satisfação do utente com o horário de atendimento da sua US» e a «Facilidade de marcação de consulta com o seu MF». As variáveis «Frequência com que o utente deixou de recorrer ao seu MF por ser dispendioso», «Frequência com que o utente deixou de fazer medicação ou outros tratamentos por ser dispendioso» e a "Frequência com que o utente deixou de fazer exames por ser dispendioso» avaliaram o «Esforço Financeiro». A «Adequabilidade» foi estudada com recurso às variáveis «Frequência com que o utente recorre a um médico no privado por não obter resposta 
com o seu MF», «Satisfação com a forma como o utente é atendido pelo seu MF» e «Confiança do utente no seu MF».

As variáveis quantitativas foram sumariadas através de estatísticas descritivas, nomeadamente, média, mediana, desvio-padrão, mínimo e máximo e as variáveis qualitativas através do cálculo de frequências absolutas (n) e relativas (\%) e intervalos de confiança a 95\%. Para avaliar cada uma das três dimensões foi criado um scoreglobal para cada dimensão a partir do cálculo da média das três variáveis dessa dimensão. Cada uma das variáveis pontuava de zero a cinco [sempre (0), quase sempre (1), muitas vezes (2), algumas vezes (3), quase nunca (4) e nunca (5)]. Assim, por exemplo, para a dimensão «Esforço financeiro", se a resposta nas três variáveis fosse "nunca" o score dessa dimensão seria de 5. Contudo, para o cálculo do score de cada dimensão houve necessidade de inverter a pontuação em algumas das variáveis para que a interpretação do score se fizesse sempre no mesmo sentido (inverteram-se as três variáveis da dimensão «Capacidade e Acomodação» e as duas últimas variáveis da dimensão «Adequabilidade»). Assim, para todas as dimensões da acessibilidade, um score mais próximo de zero correspondia a uma pior avaliação e um score mais próximo de cinco traduzia uma avaliação mais positiva. Note-se que, quando uma variável da dimensão tinha valor omisso, o score era calculado com base na média das outras duas variáveis e o score só era calculado quando pelo menos duas das três variáveis (66\%) estavam preenchidas $(\geq 50 \%)$. Se duas ou três variáveis tinham valor omisso, o score era considerado omisso. A consistência interna dos scores foi avaliada através do coeficiente de alfa de Cronbach.

Para a análise inferencial foi utilizado o teste do QuiQuadrado para testar a associação de duas variáveis qualitativas. Após a análise de normalidade, a comparação de dois grupos independentes face a uma variável quantitativa foi efetuada através do teste não paramétrico de Mann-Whitney-U, de acordo com a validação dos pressupostos inerentes a cada teste. Na comparação de três ou mais grupos independentes face a uma variável quantitativa, e uma vez que as hipóteses de normalidade e/ou de homogeneidade de variâncias não se verificaram, utilizou-se o teste não paramétrico de Kruskal-Wallis. A análise de associação entre duas variáveis quantitativas foi efetuada através do coeficiente de correlação de Spearman, uma vez que não se verificou o pressuposto de normalidade. Os testes estatísticos foram efetuados bilateralmente considerando um nível de significância de $5 \%$.

A análise estatística dos dados foi efetuada através do software estatístico IBM ${ }^{\circledR}$ SPSS $^{\circledR}$ Statistics, v. 19.

A realização deste estudo foi aprovada pelos orientadores das investigadoras, pelos coordenadores das USF Brás-Oleiro, São Pedro da Cova, Bracara Augusta e Prelada, pelos Presidentes dos Conselhos Clínicos e de Saúde dos ACeS Grande Porto II-Gondomar, Cávado I-Braga e Grande Porto V-Porto Ocidental e pela Unidade de Investigação Clínica da Administração Regional de Saúde (ARS) do Norte, tendo merecido parecer favorável por parte da Comissão de Ética da ARS Norte.

\section{RESULTADOS}

Dos 1.250 utentes selecionados, 692 (55,4\%) responderam ao questionário. Dos utentes excluídos, 446 (35,6\%) não se encontravam contactáveis, 66 (5,3\%) recusaram participar, 33 (2,6\%) mostraram-se incapazes de responder, seis $(0,5 \%)$ tinham falecido e sete $(0,6 \%)$ não correspondiam a nenhum utente identificável na lista de utentes (por algum erro não detetado na recolha inicial da população em estudo) (Figura 1).

Em média, foram realizados 115 questionários por investigadora (variação: 89 a 160 questionários). Na USF Brás-Oleiro foram aplicados $49,6 \%$ dos questionários, seguindo-se a USF Bracara Augusta com 21,7\%, a USF Prelada com $15,2 \%$ e a USF São Pedro da Cova com 13,6\%.

Em relação às características sociodemográficas dos utentes incluídos no estudo (Quadro I), a maioria dos utentes pertencia ao género feminino $(57,4 \%)$ e encontrava-se casado ou em união de facto $(65,2 \%)$. Os utentes entrevistados tinham, em média, 52 anos de idade (mediana=52 anos; variação=18 e 90 anos) e uma escolaridade média de oito anos. Relativamente à situação profissional, $323(46,7 \%)$ dos utentes que responderam estavam no ativo, 218 (31,5\%) encontravam-se reformados, 115 (16,6\%) estavam desempregados e 29 (4,2\%) eram estudantes. Em média, o agregado familiar dos utentes incluídos apresentava cerca de três elementos, variando entre um a oito elementos. A maioria dos utentes $(64,5 \%)$ apresentava um rendimento mensal líquido do agregado familiar entre os $500 € \mathrm{e}$ os $1.500 €$, em 14,6\% dos casos este era inferior a $500 €$ e em $20,2 \%$ auferiam um rendimento líquido superior a $1.500 € \mathrm{~A}$ isenção de taxa moderadora verificou-se em $49,0 \%$ dos casos. Em 


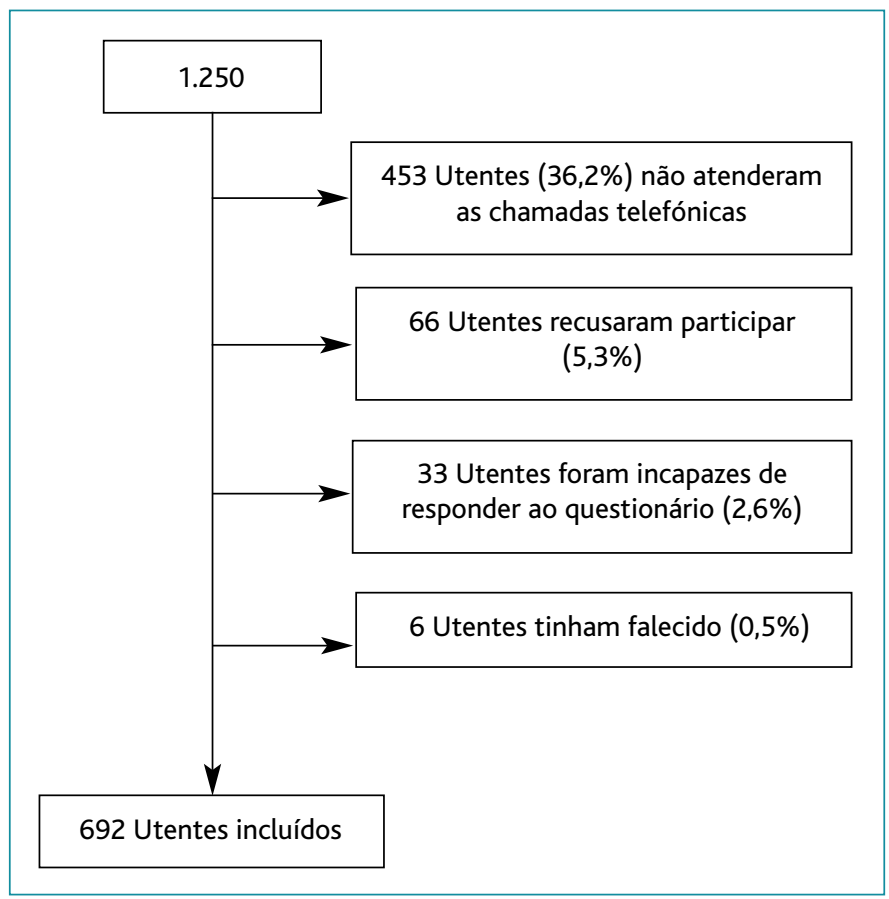

Figura 1. Utentes incluídos a partir da amostra em estudo.

$8,1 \%$ dos casos, os utentes referiram ter um estado geral de saúde "excelente", em 16,2\% "muito bom", em 33,5\% "bom", em 31,6\% "suficiente" e em 10,5\% "mau". A duração da relação do utente com o seu MF atual foi, em média, de 17 anos (mediana=17 anos; variação=1 e 45 anos).

Comparando os indivíduos excluídos e incluídos constatou-se existirem diferenças estatisticamente significativas entre os dois grupos relativamente à idade e ao género. Assim, utilizou-se o teste de Mann-Whitney- $U$ para amostras independentes para comparar os dois grupos relativamente à idade, verificando-se, em termos medianos, que os incluídos eram mais velhos que os excluídos (52 anos vs 47 anos; $p=0,003$ ). A diferença entre os dois grupos relativamente ao género foi testada através do teste Qui-quadrado, tendo-se observado que havia mais mulheres no grupo dos incluídos do que nos excluídos (57,4\% vs 50,8\%; $p=0,021$ ) (Quadro II).

Os scores mostraram uma boa consistência interna [«Capacidade e Acomodação» (alfa de Cronbach =0,663); «Esforço Financeiro» (alfa de Cronbach=0,75); «Adequabilidade» (alfa de Cronbach=0,74)]. A «Capacidade e Acomodação» obteve um score global médio de 3,91, o «Esforço Financeiro» de 4,74 e a «Adequabilidade» de 4,34.

Para comparar as USF onde foi realizado o estudo uti- lizou-se o teste de Kruskal-Wallis, tendo-se verificado a existência de diferenças estatisticamente significativas entre as USF relativamente a cada score ( $" \mathrm{Ca}-$ pacidade e Acomodação», $p<0,001$; «Esforço Financeiro», $p=0,004$; «Adequabilidade», $p<0,001$ ) (Quadro III). A USF Brás Oleiro foi a que apresentou melhor "Capacidade e Acomodação" (mediana $=4,6$; variação= 0,0 e 5,0; média $=4,1 ; D P=0,9$ ) e melhor «Adequabilidade» (mediana=5,0; variação: 0,0 e 5,0; média $=4,5 ; D P=0,8$ ). Todas as USF apresentaram igual valor mediano relativamente ao «Esforço Financeiro». No entanto, a USF Brás Oleiro foi a que apresentou melhor média relativamente a esta última dimensão (média $=4,5 ; D P=0,8$ ).

A relação entre o género, estado civil, situação profissional e as três dimensões da acessibilidade está representada no Quadro IV. O teste de Mann-Whitney-U foi utilizado para comparar o género relativamente a cada um dos scores, não se tendo observado diferenças estatisticamente significativas entre o género feminino e masculino $(p>0,05)$. Para comparar o estado civil e a situação profissional relativamente a cada score foi utilizado o teste de Kruskal-Wallis. Não se verificaram diferenças estatisticamente significativas entre o estado civil para cada um dos scores $(p>0,05)$. No entanto, constatou-se existirem diferenças estatisticamente significativas entre a situação profissional (exceto para as categorias "não aplicável" e "desconhecido" - o teste não entrou em linha de conta com estas categorias) relativamente aos scores «Capacidade e Acomodação» $(p<0,001)$ e «Adequabilidade» $(p<0,001)$. Assim, os reformados apresentaram melhor «Capacidade e Acomodação" (mediana=4,5; variação: 1,0 e 5,0). Os ativos e os estudantes tiveram pior "Adequabilidade» (mediana $=4,3$ ) em comparação com os reformados e os desempregados (mediana $=5,0$ ).

Para procurar associações entre as variáveis idade, escolaridade, número de elementos do agregado familiar e rendimento familiar e cada um dos scores foi utilizada a correlação de Spearman (Quadro V). A idade associouse quer com a «Capacidade e Acomodação», quer com a «Adequabilidade»: quanto maior a idade melhor a «Capacidade e Acomodação» $\left(r_{s}=0,123 ; p=0,001\right)$ e melhor a «Adequabilidade» $\left(r_{s}=0,124 ; p=0,001\right)$. A escolaridade mostrou correlação com os três scores: quanto menor a escolaridade melhor a «Capacidade e Acomodação» $\left(r_{s}=\right.$ $-0,165 ; p<0,001)$, maior $\mathrm{o}$ «Esforço Financeiro» $\left(r_{s}=0,140\right.$; 
$p<0,001)$ e melhor a "Adequabilidade» $\left(r_{s}=\right.$ $0,253 ; p<0,001)$. O número de elementos do agregado familiar, assim como o rendimento familiar, também se correlacionaram com os scores obtidos em cada uma das três dimensões: quanto menor o agregado familiar melhor a «Capacidade e Adequabilidade» $\left(r_{s}=\right.$ $0,150 ; p<0,001)$, menor $\mathrm{o}$ «Esforço Financeiro» $\left(r_{s}=-0,103 ; p=0,007\right)$ e melhor a «Adequabilidade» $\left(r_{s}=-0,166 ; p<0,001\right)$; quanto menor o rendimento mensal melhor a «Capacidade e Acomodação» $\left(r_{s}=-0,141 ; p<0,001\right)$ e melhor a «Adequabilidade» $\left(r_{s}=-0,295 ; p<0,001\right) \mathrm{e}$, em sentido inverso, quanto maior o rendimento mensal menor o «Esforço Financeiro" $\left(r_{s}=0,173\right.$; $p<0,001)$. Assim, ainda que baixas, as correlações mostraram-se estatisticamente significativas para estas quatro variáveis relativamente a alguns dos scores.

Na tentativa de esclarecer que influência a isenção de taxas moderadoras poderia ter na pontuação destes scores, o teste Mann-Whit$n e y-U$ foi novamente utilizado, desta vez para comparar o estado de isenção de taxa moderadora (isento vsnão isento) relativamente a cada score (Quadro IV). Assim, no mesmo sentido que o verificado para o rendimento mensal, observou-se que existiam diferenças estatisticamente significativas entre estar ou não isento relativamente a cada um dos scores («Capacidade e Acomodação», p=0,036; «Esforço Financeiro», $\mathrm{p}<0,001 \mathrm{e}$ «Adequabilidade», $\mathrm{p}=0,005)$. Os isentos e os não isentos apresentaram o mesmo valor mediano na "Capacidade e Acomodação» (mediana $=4,0$ ) e no «Esforço Financeiro» (mediana $=5,0$ ). No entanto, em termos médios, os isentos apresentaram melhor "Capacidade e Acomodação" (média $=4,0 ; D P=1,1$ ) e os não isentos apresentaram menor «Esforço Financeiro» (média $=4,8 ; D P=0,5$ ). Os isentos foram os que apresentaram melhor «Adequabilidade» (mediana=5,0; variação: 1,0 e 5,0).

Recorreu-se ainda à correlação de Spearman para analisar a associação quer do estado geral de saúde, quer a duração da relação do utente com o seu médico de família com cada score (Quadro V). Encontraram-se correlações esta-

\begin{tabular}{|c|c|}
\hline & $\begin{array}{c}\text { Total } \\
(n=692)\end{array}$ \\
\hline \multicolumn{2}{|l|}{ Género, $n(\%)$} \\
\hline Feminino & $397(57,4)$ \\
\hline Masculino & $295(42,6)$ \\
\hline \multicolumn{2}{|l|}{ Estado civil, $n$ (\%) } \\
\hline Solteiro(a) & $142(20,5)$ \\
\hline Casado(a)/União de facto & $451(65,2)$ \\
\hline Divorciado(a) & $41(5,9)$ \\
\hline Viúvo(a) & $58(8,4)$ \\
\hline \multicolumn{2}{|l|}{ Situação profissional* ${ }^{*} n(\%)$} \\
\hline Ativo(a) & $323(46,7)$ \\
\hline Desempregado(a) & $115(16,6)$ \\
\hline Reformado(a) & $218(31,5)$ \\
\hline Estudante & $29(4,2)$ \\
\hline \multicolumn{2}{|l|}{ Rendimento mensal líquido do agregado familiar ${ }^{\dagger}, n(\%)$} \\
\hline$<500$ & $101(14,6)$ \\
\hline $500-1000$ & $276(39,9)$ \\
\hline $1000-1500$ & $170(24,6)$ \\
\hline $1500-2000$ & $58(8,4)$ \\
\hline$>2000$ & $82(11,8)$ \\
\hline \multicolumn{2}{|l|}{ Isenção, $n$ (\%) } \\
\hline Isento & $339(49,0)$ \\
\hline Não isento & $353(51,0)$ \\
\hline \multicolumn{2}{|l|}{ Estado geral de saúde, $n$ (\%) } \\
\hline Excelente & $56(8,1)$ \\
\hline Muito boa & $112(16,2)$ \\
\hline Boa & $232(33,5)$ \\
\hline Suficiente & $219(31,6)$ \\
\hline Má & $73(10,5)$ \\
\hline \multicolumn{2}{|l|}{ Idade (anos) } \\
\hline$N$ & 692 \\
\hline média \pm DP & $51,5 \pm 16,7$ \\
\hline \multicolumn{2}{|l|}{ Escolaridade (anos) } \\
\hline$N$ & 692 \\
\hline média \pm DP & $8,4 \pm 4,7$ \\
\hline \multicolumn{2}{|l|}{ N. ${ }^{\circ}$ elementos agregado familiar ${ }^{\ddagger}$} \\
\hline$N$ & 690 \\
\hline média \pm DP & $2,8 \pm 1,1$ \\
\hline \multicolumn{2}{|l|}{ Duração da relação com o Médico de Família§ } \\
\hline$N$ & 522 \\
\hline média \pm DP & $17,3 \pm 10,4$ \\
\hline
\end{tabular}

* Sete utentes não sabiam a sua situação profissional ou não se enquadravam em nenhuma das opções.

$\dagger$ Cinco utentes recusaram responder a esta questão.

₹ Dois utentes recusaram responder a esta questão.

$\S$ Cento e setenta utentes não sabiam há quanto tempo tinham o mesmo médico de família. 


\begin{tabular}{|c|c|c|c|}
\hline \multicolumn{4}{|c|}{$\begin{array}{l}\text { QUADRO II. Comparação entre os utentes incluídos e } \\
\text { excluídos do estudo relativamente ao género e idade }\end{array}$} \\
\hline & Incluídos & Excluídos & Valor $p$ \\
\hline \multicolumn{4}{|l|}{ Género, $n(\%)$} \\
\hline Feminino & $397(57,4)$ & $280(50,8)$ & $0,021^{\text {a) }}$ \\
\hline Masculino & $295(42,6)$ & $271(49,2)$ & \\
\hline \multicolumn{4}{|l|}{ Idade (anos) } \\
\hline$N$ & 692 & 551 & $0,003^{b)}$ \\
\hline mediana (min - máx) & $52(18$ e 90$)$ & 47 (19 e 102) & \\
\hline
\end{tabular}

a) Teste do Qui-Quadrado.

b) Teste de Mann-Whitney.

tisticamente significativas, ainda que baixas, de ambas as variáveis relativamente aos scores de «Capacidade e Acomodação» e de "Adequabilidade». Assim, quanto pior a perceção do estado geral de saúde, melhor a «Capacidade e Acomodação» $(r=0,076 ; p=0,046)$ e melhor a «Adequabilidade» $(r=0,143 ; p<0,001)$; quanto maior a duração da relação do utente com o seu médico de família, melhor a "Capacidade e Acomodação» $\left(r_{s}=0,153\right.$; $p<0,001)$ e melhor a "Adequabilidade» $\left(r_{s}=0,212\right.$; $p<0,001)$.

\section{DISCUSSÃO}

Neste estudo, a acessibilidade foi avaliada através de parâmetros que surgem após a perceção, por parte dos utentes, da necessidade de cuidados de saúde: a «Capacidade e Acomodação», que reflete sobretudo a capacidade das US oferecerem serviços em quantidade suficiente e a facilidade com que os utentes podem recorrer a esses serviços; o «Esforço Financeiro», que exprime os custos associados aos cuidados prestados; e a «Adequabilidade», que expressa a qualidade da relação médico-doente e a perceção de qualidade dos cuidados providenciados no serviço de saúde.

$\mathrm{Na}$ ausência de um instrumento consistente e validado que avaliasse o nível socioeconómico na sua globalidade, utilizaram-se alguns parâmetros individuais para o determinar de forma indireta, como o grau de escolaridade, o número de elementos e o rendimento mensal do agregado familiar, a situação ocupacional e o estado de isenção. Posteriormente procurou-se demonstrar de que forma estariam relacionados ou não com a acessibilidade aos CSP nesta população da região norte de Portugal.

Relativamente à escolaridade e ao rendimento mensal do agregado familiar verificou-se que, quanto menor o número de anos frequentados na escola pelos utentes e menor o seu rendimento, melhor a sua acessibilidade aos CSP em todos os aspetos, exceto no que diz respeito ao «Esforço Financeiro». Assim, assumindo que os indivíduos com menos anos de escolaridade e menor rendimento mensal pertencem a um nível socioeconómico mais desfavorecido, estes apresentaram, como seria de esperar, mais dificuldade em comprar medicação, efetuar tratamentos, realizar exames complementares de diagnóstico e suportar os custos inerentes à visita médica (transporte, perda de remuneração por absentismo, taxa moderadora). No entanto, em contraste, estes indivíduos referiram uma maior facilidade em deslocar-se e aceder a uma consulta com o seu MF, assim como manifestaram uma maior satisfação com este, em comparação com os indivíduos de maior escolaridade e rendimento mensal, ou seja, pertencentes a um nível socio-

QUADRO III. Comparação entre as USFs relativamente a cada dimensão da Acessibilidade

\begin{tabular}{l|c|c|c|c|c|c} 
& $\boldsymbol{n}$ & $\begin{array}{c}\text { "Capacidade e acomodação" } \\
\text { mediana (min-max) }\end{array}$ & $\boldsymbol{n}$ & $\begin{array}{c}\text { "Esforço financeiro" } \\
\text { mediana (min-max) }\end{array}$ & $\begin{array}{c}\boldsymbol{n} \\
\text { "Adequação" } \\
\text { mediana (min-max) }\end{array}$ \\
\hline USF Brás-Oleiro & 342 & $4,6(0,0-5,0)$ & 341 & $5,0(2,0-5,0)$ & 342 & $5,0(0,0-5,0)$ \\
\hline USF Bracara Augusta & 150 & $3,3(1,0-5,0)$ & 150 & $5,0(3,0-5,0)$ & 150 & $3,8(1,0-5,0)$ \\
\hline USF Prelada & 105 & $4,3(1,0-5,0)$ & 105 & $5,0(1,0-5,0)$ & 105 & $4,7(1,0-5,0)$ \\
\hline USF S. Pedro da Cova & 94 & $4,0(2,0-5,0)$ & 94 & $5,0(3,0-5,0)$ & 94 & $5,0(2,0-5,0)$ \\
\hline Valor $\boldsymbol{P}^{\text {a) }}$ & & $\boldsymbol{p}<0,001$ & & $\boldsymbol{p = 0 , 0 0 4}$ & & $\boldsymbol{p < 0 , 0 0 1}$ \\
\hline
\end{tabular}

$n-\mathrm{N} .^{\circ}$ total de utentes em que foi calculado o score relativamente a cada dimensão da acessibilidade.

a) Teste de Kruskall-Wallis. 


\begin{tabular}{|c|c|c|c|c|c|c|}
\hline & $n$ & $\begin{array}{c}\text { "Capacidade e acomodação" } \\
\text { mediana (min-max) }\end{array}$ & $n$ & $\begin{array}{l}\text { "Esforço financeiro" } \\
\text { mediana (min-max) }\end{array}$ & $n$ & $\begin{array}{c}\text { "Adequação" } \\
\text { mediana (min-max) }\end{array}$ \\
\hline \multicolumn{7}{|l|}{ Género } \\
\hline Feminino & 396 & $4,0(0,0-5,0)$ & 396 & $5,0(1,0-5,0)$ & 396 & $4,7(1,0-5,0)$ \\
\hline Masculino & 295 & $4,0(1,0-5,0)$ & 294 & $5,0(1,0-5,0)$ & 295 & $4,7(0,0-5,0)$ \\
\hline Valor $p^{\text {a) }}$ & & $p=0,315$ & & $p=0,279$ & & $p=0,638$ \\
\hline \multicolumn{7}{|l|}{ Estado civil } \\
\hline Solteiro(a) & 141 & $4,0(1,0-5,0)$ & 141 & $5,0(3,3-5,0)$ & 141 & $4,7(0,0-5,0)$ \\
\hline $\begin{array}{l}\text { Casado(a)/União } \\
\text { de facto }\end{array}$ & 451 & $4,0(1,0-5,0)$ & 450 & $5,0(1,0-5,0)$ & 451 & $4,7(1,0-5,0)$ \\
\hline Divorciado(a) & 41 & $4,3(0,0-5,0)$ & 41 & $5,0(3,0-5,0)$ & 41 & $5,0(2,7-5,0)$ \\
\hline Viúvo(a) & 58 & $4,0(1,0-5,0)$ & 58 & $5,0(2,3-5,0)$ & 58 & $5,0(1,0-5,0)$ \\
\hline Valor $\boldsymbol{p}^{\mathrm{b})}$ & & $p=0,230$ & & $p=0,124$ & & $p=0,100$ \\
\hline \multicolumn{7}{|l|}{ Situação profissional } \\
\hline Ativo(a) & 323 & $4,0(1,0-5,0)$ & 323 & $5,0(2,7-5,0)$ & 322 & $4,3(0,0-5,0)$ \\
\hline Desempregado(a) & 115 & $4,3(0,0-5,0)$ & 114 & $5,0(1,0-5,0)$ & 115 & $5,0(1,7-5,0)$ \\
\hline Reformado(a) & 218 & $4,5(1,0-5,0)$ & 218 & $5,0(2,3-5,0)$ & 218 & $5,0(1,0-5,0)$ \\
\hline Estudante & 28 & $4,0(2,0-5,0)$ & 28 & $5,0(4,0-5,0)$ & 28 & $4,3(2,3-5,0)$ \\
\hline Valor $p^{\text {b) }}$ & & $p<0,001$ & & $p=0,170$ & & $p<0,001$ \\
\hline \multicolumn{7}{|l|}{ Isenção } \\
\hline Isento & 339 & $4,0(0,0-5,0)$ & 338 & $5,0(1,0-5,0)$ & 339 & $5,0(1,0-5,0)$ \\
\hline Não isento & 352 & $4,0(1,0-5,0)$ & 352 & $5,0(2,3-5,0)$ & 352 & $4,3(0,0-5,0)$ \\
\hline Valor $p^{\text {a) }}$ & & $p=0,036$ & & $p=0,005$ & & $p<0,001$ \\
\hline
\end{tabular}

$n-\mathrm{N} .{ }^{\circ}$ total de utentes em que foi calculado o score relativamente a cada dimensão da acessibilidade.

a) Teste de Mann-Whitney.

b) Teste de Kruskall-Wallis.

económico mais favorecido. Por outro lado, grande parte da população tem seguros de saúde privado para além do serviço público e a frequência com que acede ao serviço de saúde privado pode variar de acordo com o nível educacional. ${ }^{3}$ Assim, poderá especular-se que os indivíduos com nível de escolaridade mais elevado, e que poderão apresentar consequentemente maiores rendimentos, possam recorrer mais aos serviços de saúde privados, diminuindo desta forma a acessibilidade destes ao serviço público.

Em termos de situação profissional, os reformados foram o grupo que teve uma melhor «Capacidade e Acomodação». O facto de estes poderem apresentar uma maior disponibilidade ao longo do dia poderá justificar uma maior facilidade em se deslocarem à sua US, satisfação com o seu horário de funcionamento e flexibilidade na marcação de consultas. Em contrapartida, os indiví- duos ativos e os estudantes evidenciaram pior «Adequabilidade», isto é, mostraram-se menos satisfeitos com os cuidados de saúde prestados pelo seu MF, assim como com a relação estabelecida com este. Considerando que estes indivíduos serão, à partida, mais jovens e terão uma menor disponibilidade diária devido à sua atividade profissional, é provável que sejam mais exigentes e tenham necessidade de resolver os seus problemas de saúde de forma mais célere e efetiva, pois o tempo de que dispõem é menor em comparação com os utentes desempregados e reformados. Além disso, os utentes estudantes e ativos recorrem, à partida, menos vezes ao médico, condicionando um menor contacto com este e, por isso, menos oportunidades de estabelecimento de uma relação de confiança com este, podendo justificar uma menor adequabilidade dos cuidados. $\mathrm{O}$ esforço financeiro não afetou a acessibilidade em nenhum destes grupos. 


\begin{tabular}{|c|c|c|c|c|c|c|}
\hline & $n$ & "Capacidade e acomodação" & $n$ & "Esforço financeiro" & $n$ & "Adequação" \\
\hline Idade & 691 & $\begin{array}{l}r_{s}=0,123 \\
p=0,001\end{array}$ & 690 & $\begin{array}{l}r_{s}=0,008 \\
p=0,841\end{array}$ & 691 & $\begin{array}{l}r_{s}=0,124 \\
p=0,001\end{array}$ \\
\hline Escolaridade & 691 & $\begin{array}{r}r_{s}=-0,165 \\
p<0,001\end{array}$ & 690 & $\begin{array}{l}r_{s}=0,140 \\
p<0,001\end{array}$ & 691 & $\begin{array}{r}r_{s}=-0,253 \\
p<0,001\end{array}$ \\
\hline $\begin{array}{l}\text { N. }{ }^{\circ} \text { elementos do } \\
\text { agregado familiar }\end{array}$ & 689 & $\begin{array}{r}r_{s}=-0,150 \\
p<0,001\end{array}$ & 689 & $\begin{array}{r}r_{s}=-0,103 \\
p=0,007\end{array}$ & 689 & $\begin{array}{r}r_{s}=-0,166 \\
p<0,001\end{array}$ \\
\hline $\begin{array}{l}\text { Rendimento mensal } \\
\text { líquido do agregado } \\
\text { familiar }\end{array}$ & 686 & $\begin{array}{r}r_{s}=-0,141 \\
p<0,001\end{array}$ & 685 & $\begin{array}{l}r_{s}=0,173 \\
p<0,001\end{array}$ & 686 & $\begin{array}{r}r_{s}=-0,295 \\
p<0,001\end{array}$ \\
\hline $\begin{array}{l}\text { Duração da relação do } \\
\text { utente com o seu MF }\end{array}$ & 522 & $\begin{array}{l}r_{s}=0,153 \\
p<0,001\end{array}$ & 521 & $\begin{array}{l}r_{s}=0,046 \\
p=0,290\end{array}$ & 522 & $\begin{array}{l}r_{s}=0,212 \\
p<0,001\end{array}$ \\
\hline Estado geral de saúde & 691 & $\begin{array}{l}r_{s}=0,076 \\
p=0,046\end{array}$ & 690 & $\begin{array}{r}r_{s}=-0,062 \\
p=0,104\end{array}$ & 691 & $\begin{array}{l}r_{s}=0,143 \\
p<0,001\end{array}$ \\
\hline
\end{tabular}

Legenda: MF - Médico de Família; $\boldsymbol{n}$ - N. ${ }^{\circ}$ total de utentes em que foi calculado o score relativamente a cada dimensão da acessibilidade; $r_{s}-$ Coeficiente de correlação de Spearman.

Relativamente ao tamanho do agregado familiar verificou-se que, quanto menor o número de elementos, melhor a acessibilidade, em todos os parâmetros. A relação entre o número de elementos do agregado familiar e o nível socioeconómico poderá ser mais difícil de estabelecer. À primeira vista, poderia depreender-se que quanto menor o agregado familiar maior a disponibilidade financeira por elemento e, de uma forma global, melhor seria o nível socioeconómico da família. Desta forma, e contrariando o que tem vindo a ser estabelecido, um menor agregado familiar (melhor nível socioeconómico) estaria associado a uma maior acessibilidade aos cuidados de saúde primários. Numa outra perspetiva, famílias abastadas poderão ter mais filhos e, com medidas de planeamento familiar, famílias de baixos rendimentos poderão escolher ter menos. Assim, um menor agregado familiar, associado agora a um nível socioeconómico mais baixo, estaria associado a melhor acessibilidade. Desta forma, a relação do tamanho do agregado com o nível socioeconómico carece de suporte bibliográfico e necessita de ser melhor estabelecida em trabalhos futuros.

Os indivíduos isentos de taxa moderadora revelaram melhor «Capacidade e Acomodação» e "Adequabilidade», enquanto os não isentos mostraram menor «Esforço Financeiro». Os isentos são provavelmente indivíduos com menor rendimento económico, desempregados ou reformados, pelo que terão, à partida, mais facilidade em aceder ao seu MF. Além disso, como recorrem mais frequentemente ao serviço público em detrimento do privado, a probabilidade de estarem mais satisfeitos e confiarem no seu MF é maior. De destacar que a isenção de taxa moderadora se verificou em $49 \%$ dos casos, o que é sensivelmente semelhante à média nacional (53\%). ${ }^{\text {? }}$

A perceção do estado geral de saúde bem como a duração da relação com o MF não demonstraram associação com o «Esforço Financeiro». Por outro lado, uma pior perceção de estado de saúde e uma maior duração daquela relação associaram-se a uma maior acessibilidade no que diz respeito à «Capacidade e Acomodação» e à «Adequabilidade». Mais acessibilidade para os que têm pior perceção de estado de saúde vai de encontro ao princípio de justiça distributiva que se refere à distribuição justa, equitativa e apropriada dos recursos de saúde, isto é, "dar mais a quem mais precisa". ${ }^{8}$ Paralelamente, uma relação mais longa providencia maior conhecimento mútuo e solidificação da relação de confiança médico-utente.

Todas as unidades de saúde mostraram, de uma forma global, boa acessibilidade, destacando-se a USF Brás Oleiro nos parâmetros «Capacidade e Acomodação» e «Adequabilidade». Isto pode dever-se, em parte, à localização geográfica desta unidade, pois situa-se numa região mais periférica e, portanto, com menos unidades 
privadas de saúde, levando ao recurso mais assíduo dos doentes aos CSP.

Sobre a utilização de subsistemas e serviços privados, em Portugal existem mais de 2.500.000 habitantes com subsistemas ou com seguros de saúde (2010) e uma diversidade de prestadores de cuidados de saúde. Do total da população residente em Portugal, $81,1 \%$ recorre ao SNS, $10,1 \%$ tem acesso à Assistência na Doença aos Servidores Civis do Estado (ADSE), 1,3\% não utiliza nenhum subsistema de saúde, 7,5\% utiliza outros subsistemas e $10,5 \%$ possui seguros de saúde privados. ${ }^{9}$ Neste contexto, inquirir os utentes sobre a posse de um seguro de saúde poderia ser útil; contudo, não seria ético.

Analisando os resultados, depreende-se que a taxa de resposta ficou aquém do esperado, sendo o principal motivo os utentes não se encontrarem contactáveis. Deveuse, provavelmente, à não atualização dos contactos telefónicos da lista de utentes e ao facto de os contactos terem sido efetuados unicamente durante o horário de funcionamento das USF (8h-20h) que poderá ter coincidido com o horário laboral de grande parte da população ativa. Outra limitação do estudo foi a criação de um questionário não validado para determinação da acessibilidade. $\mathrm{O}$ facto de uma das USF estar quantitativamente mais representada do que as restantes poderá ter influenciado os resultados, enviesando as conclusões retiradas. Por outro lado, verificou-se que existiam diferenças entre os excluídos e os incluídos - os utentes incluídos eram, em média, mais velhos do que os excluídos, provavelmente por apresentarem maior disponibilidade de tempo, uma vez que uma grande parte desta população se encontra inativa -, criando problemas na interpretação dos resultados e extrapolação dos dados obtidos para a população geral.

Em relação à avaliação do estado geral de saúde, deve salientar-se que esta é subjetiva, sendo autorreportada e dependendo unicamente da perceção do utente. No entanto, à semelhança deste estudo, noutros ${ }^{2}$ a avaliação do estado de saúde era realizada apenas através de uma simples questão ou através de uma combinação de questões sobre incapacidade e perceção do estado de saúde. Em última análise, mais importante do que conhecer o estado geral de saúde, é saber a perceção que o próprio tem do seu estado de saúde. Adicionalmente, e de acordo com a literatura, ${ }^{10}$ a probabilidade de ser observado por um MF parece estar distribuída de acordo com a necessidade e não é muito influenciada pelo rendimento.
Contudo, no presente estudo não foi feito o ajuste para o estado geral de saúde, pelo que a relação entre o nível socioeconómico e a acessibilidade poderá estar enviesada.

De acordo com estudos prévios, a frequência de consultas/ano poderá influenciar a acessibilidade; contudo, este não foi um dos parâmetros avaliado. Poderão existir erros na informação obtida através do sistema informático, nomeadamente relativa à data do último contacto. A amostragem telefónica poderá ter excluído utentes que não apresentassem contacto telefónico registado ou atualizado no sistema informático. Este estudo também não avaliou de forma precisa os custos indiretos totais no acesso aos cuidados de saúde.

Em Portugal, um estudo realizado pela Entidade Reguladora de Saúde em $2009^{1}$ procurou avaliar vários parâmetros da acessibilidade aos CSP. Relativamente à dimensão "Capacidade e Acomodação» (naquele estudo representadas por «Proximidade» e «Adequabilidade») concluiu-se que os CSP se encontram próximos dos utentes, já que a população residente fora do alcance de um centro de saúde (a mais de 30 minutos) não chega a representar $0,03 \%$ da população. Por outro lado, as avaliações positivas em relação aos horários de funcionamento das consultas e facilidade da sua marcação foram mais predominantes do que as avaliações negativas. Contudo, o estudo supracitado apenas avaliou descritivamente os diversos parâmetros da acessibilidade, mas não a relacionou com o nível socioeconómico, ao contrário do presente estudo, enfatizando a inovação do mesmo.

A avaliação detalhada do nível socioeconómico, a elaboração de um questionário original e a realização de um questionário telefónico a um elevado número de indivíduos são outras mais-valias deste estudo.

Globalmente, o presente estudo permitiu mostrar que um nível socioeconómico mais desfavorecido e uma pior perceção do estado de saúde estavam correlacionados com uma maior acessibilidade, fundamentalmente no que diz respeito à «Capacidade e Acomodação» e «Adequabilidade» para a população analisada.

Dada a escassez de estudos realizados noutras populações que associem a acessibilidade e o nível socioeconómico é fundamental que se realizem, no futuro, estudos semelhantes.

\section{REFERÊNCIAS BIBLIOGRÁFICAS}

1. Entidade Reguladora da Saúde. Estudo do acesso aos cuidados de saú- 
de primários do SNS [Internet]. Porto: ERS; 2009. Available from: https://www.ers.pt/uploads/writer_file/document/86/ERS_-_Estudo_do_Acesso_aos_Cuidados_de_Saude_Primarios_-_Relatorio.pdf

2. Hanratty $B$, Zhang $T$, Whitehead M. How close have universal health systems come to achieving equity in use of curative services? A systematic review. Int J Health Serv. 2007;37(1):89-109.

3. Lostao L, Regidor E, Geyer S, Aïach P. Patient cost sharing and physician visits by socioeconomic position: findings in three Western European countries. J Epidemiol Community Health. 2007;61(5):416-20.

4. Penchansky R, Thomas JW. The concept of access: definition and relationship to consumer satisfaction. Med Care. 1981;19(2):127-40.

5. Levesque JF, Harris MF, Russell G. Patient-centred access to health care: conceptualising access at the interface of health systems and populations. Int J Equity Health. 2013;12:18.

6. Safran DG, Kosinski M, Tarlov AR, Rogers WH, Taira DH, Lieberman N, et al. The Primary Care Assessment Survey: tests of data quality and measurement performance. Med Care. 1998;36(5):728-39.

7. Administração Central do Sistema de Saúde. Taxas moderadoras [Internet]. Lisboa: Ministério da Saúde; 2016. Available from: http://www.acss.min-saude.pt/2016/09/15/taxas-moderadoras/
8. Nunes R. Regulação na saúde. $3^{\mathrm{a}}$ ed. Porto:Vida Económica; 2014. ISBN 9789897680687.

9. Direção-Geral da Saúde. Plano nacional de saúde, 2012-2016. Lisboa: DGS; 2013.

10. Van Doorslaer E, Masseria C, Koolman X. Inequalities in access to medical care by income in developed countries. CMAJ. 2006;174(2):17783.

\section{CONFLITO DE INTERESSES}

As autoras declaram não ter conflitos de interesses.

\section{ENDEREÇO PARA CORRESPONDÊNCIA}

Vânia Teixeira

R. Serafim da Silva Lopes, $32-2^{\circ} \mathrm{dto}$

4465-261 São Mamede de Infesta

E-mail: vaniafteixeira@gmail.com

Recebido em 24-07-2015

Aceite para publicação em 22-08-2016

\section{ABSTRACT}

\section{SOCIOECONOMIC STATUS AND ACCESS TO PRIMARY HEALTH CARE: A CROSS-SECTIONAL STUDY IN FOUR HEALTH UNITS IN NORTHERN PORTUGAL}

Objectives: To examine the association between socioeconomic status and access to Primary Health Care in four Family Health Units (FHU) in the north of Portugal, in the dimensions of health care needs perceived by the patient, namely availability and accommodation, affordability and appropriateness.

Type of Study: Cross-sectional study.

Setting: Brás-Oleiro FHU, São Pedro da Cova FHU, Bracara Augusta FHU, and Prelada FHU.

Participants: Adult patients (18 years of age or older) registered in these health units for more than 12 months, on the lists of one of four family medicine residency trainers, with at least one contact in the last 24 months.

Methods: A telephone survey was conducted between April and December 2014. Each of the dimensions of accessibility was evaluated by a global score, which was related to the socioeconomic status of the population. We used the Qui-Square, Mann-Whitney, Kruskal-Wallis and Spearman correlation to test associations.

Results: Of the 1,250 selected patients, 692 (55.4\%) answered the questionnaire. We found statistically significant differences between health units for each score $(p<0.05)$. Education, income, family size, and health fee exemption status were significantly correlated with all scores. Age, professional status, global health status and the duration of the relationship between the patient and the family doctor were significantly correlated with the scores for "availability and accommodation" $(p<0.05)$ and "appropriateness" $(p<0.05)$. Conclusions: In this population, a disadvantaged socioeconomic status and a worse health status were correlated with greater accessibility to health care, especially regarding «Availability and accommodation» and «Appropriateness».

Keywords: Primary Health Care; Socioeconomic Status; Accessibility. 